\title{
Surface-emitting, distributed-feedback diode lasers with uniform near-field intensity profile
}

\author{
James Lopez, Masoud Kasraian, and Dan Botez \\ Department of Electrical and Computer Engineering, University of Wisconsin-Madison, \\ Madison, Wisconsin 53706-1691
}

(Received 16 June 1998; accepted for publication 15 August 1998)

\begin{abstract}
Theoretical analysis of second-order surface-emitting, complex-coupled distributed feedback diode lasers with first-order distributed Bragg reflectors (DBR) is presented. The DBR reflectors are shown to insure simultaneous operation in a virtually uniform near-field profile with high efficiency and adequate intermodal discrimination. Such devices display symmetric-mode (single-lobe) surface emission with relatively high external differential quantum efficiency (30\%), low gain threshold $\left(18 \mathrm{~cm}^{-1}\right)$, and $<8 \%$ near-field intensity profile variations (in the longitudinal direction). The devices have the potential to provide $>100 \mathrm{~mW}$ of stable, single-mode cw power, significantly higher than it is possible with vertical-cavity surface-emitting lasers. It is also shown that the device studied here can be combined with a resonant optical waveguide array device to produce a $2 \mathrm{D}$ uniform near-field surface-emitting source capable of providing greater than $1 \mathrm{~W} c \mathrm{cw}$ power in a stable, single-lobed beam. (C) 1998 American Institute of Physics. [S0003-6951(98)01542-3]
\end{abstract}

Complex-coupled, distributed-feedback (CC-DFB) edgeemitting lasers have recently received considerable theoretical and experimental attention as potential light sources in advanced optical-communication systems. ${ }^{1-6}$ We have previously reported the theoretical analysis of antiphase-type $e^{7,8}$ (i.e., excess gain preferentially placed in the low-index regions) second-order, surface-emitting (SE)-CC-DFB lasers. Such devices can be made to fundamentally favor operation in a single-lobed beam that is normal to the surface. Although ridge-guided devices of this type should be capable of providing $50-100 \mathrm{~mW} \mathrm{cw}$ power, they are unsuitable for high power single-mode applications, since they have highly nonuniform near-field intensity profiles ${ }^{7}$ which make them susceptible to multimode operation due to longitudinal gain spatial hole burning (GSHB) at high drive levels above threshold. Here we show that by integrating first-order distributed Bragg reflectors (DBR) at the ends of the SE-CCDFB structure, surface-emitting devices can be made to lase with both high external differential quantum efficiencies, $\eta_{D}$, as well as highly uniform near-field intensity profiles. That is, a design is presented for high-power $(>100 \mathrm{~mW})$ stable, single-mode operation from surface-emitting diode lasers.

Figure 1 schematically depicts a second-order SE-CCDFB with first-order DBRs. Surface emission occurs only in

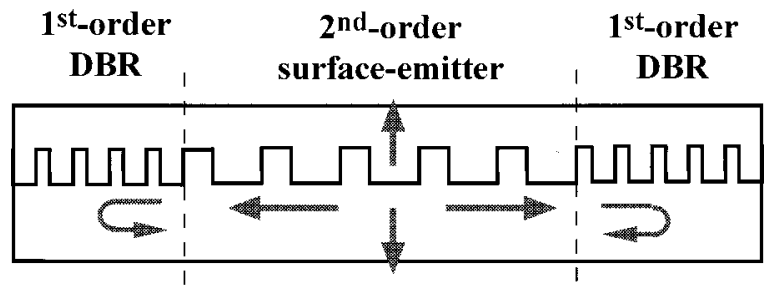

FIG. 1. Schematic diagram of a second-order surface-emitting complexcoupled (SE-CC) DFB laser structure terminated with first-order DBR reflectors. the second-order region, while the first-order DBRs serve as terminations for the SE-CC-DFB. We show that such devices can lase in a single-lobed beam pattern with gain thresholds of $\sim 18 \mathrm{~cm}^{-1}$ and an $\eta_{D}$ value of $30 \%$, while maintaining a virtually uniform near-field profile. Furthermore, it can be inferred that by combining this surface-emitting device with a resonant optical waveguide (ROW) array one can create a two-dimensional surface-emitting laser capable of providing greater than $1 \mathrm{~W}$ cw single-mode power in a stable, singlelobed beam.

The longitudinal cross section of the device studied is shown schematically in Fig. 2. The design utilizes a multiquantum-well active region ${ }^{8}$ (at $\lambda_{0}=0.98 \mu \mathrm{m}$ ). The grating structure is a rectangular grating chemically etched into the $p$-InGaP cladding layer with GaAs regrown on top and in direct contact with $\mathrm{Au}$, the electrical-contact metal. During the regrowth of GaAs, InGaAs quantum wells, which are absorbing at $980 \mathrm{~nm}$, are grown in the troughs of the InGaP grating. These absorbing InGaAs quantum wells provide the necessary modal gain modulation for favoring the symmetric mode over the antisymmetric one. ${ }^{8}$ We have already demonstrated the feasibility of such a structure by etching a $0.1 \mu \mathrm{m}$ deep grating in $\mathrm{InGaP}$ and regrowing 250

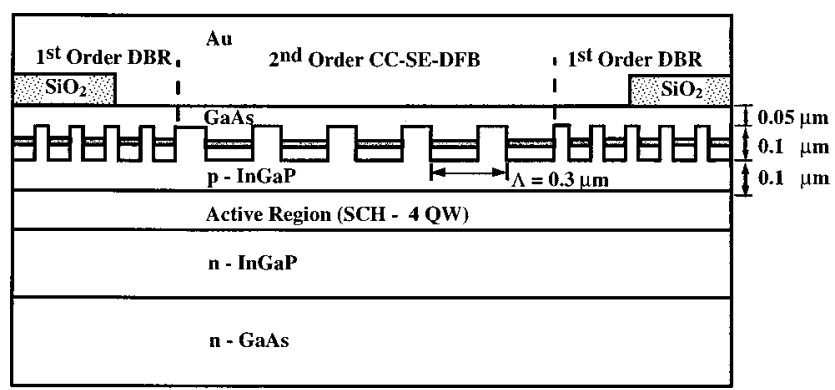

FIG. 2. Schematic longitudinal cross section of the device studied. The grating is semiconductor based, with periodically embedded GaAs with InGaAs quantum wells for absorption. The active region is described in Ref. 8. The second-order grating length is $500 \mu \mathrm{m}$. 
$\AA \mathrm{GaAs} / 150 \AA \mathrm{InGaAs} / 1100 \AA \mathrm{GaAs}$ over the grating. Transmission electron microscopy photographs show that $100-150 \AA$ thick InGaAs quantum wells are curved but well defined in the troughs of the InGaP grating. Furthermore, the upper layer of GaAs planarizes at $\sim 0.05 \mu \mathrm{m}$ above the grating peaks, thus providing a flat reflecting surface, upon metallization with $\mathrm{Au}$, that insures effective collection of all light outcoupled by the grating. The second-order SE-CCDFB is terminated with first-order DBRs. Combinations of first- and second-order gratings have been previously reported, ${ }^{9}$ with first-order DBRs used for laser oscillation and a second-order grating used as a passive outcoupler. Such a combination can be fabricated in a one-step process using direct-write $e$-beam ${ }^{9}$ or x-ray lithography. Saturation of the absorbing regions at high drive levels is not of concern for two reasons: (1) the quantum-well absorber is curved and in close proximity $(0.05-0.1 \mu \mathrm{m})$ to the metal contact, thus allowing for easy replacement of the carriers used in absorption; and (2) other designs ${ }^{7,8}$ involve metal gratings which by their nature are nonsaturable absorbers.

For the case where no first-order DBR reflectors are present, the numerical method developed by Noll and Macomber ${ }^{10}$ was used to find the coupling coefficient, $\kappa$ $=j \kappa_{i}+\kappa_{g}$, where $\kappa_{i}$ and $\kappa_{g}$ are the gain and index coupling coefficients, respectively, for an infinitely long grating, and then the coupled-mode theory (CMT) was used to solve for the modes of a finite structure. The boundary conditions used for the CMT are found by assuming that no light is entering the grating longitudinally from either end. The analysis for the case when DBR reflectors are present is done the same way, only using different boundary conditions. The boundaries are assumed to be the two interfaces of the second- and first-order gratings. The amount of light entering at each second-order grating end is simply the amount of field at the end of the second-order grating multiplied by the field amplitude reflectivity of a DBR reflector, $\rho$. Because the active region and the absorbing grating extend into the DBR regions, there is absorption which limits the maximum achievable $\rho$ value to $\approx 80 \%-85 \%$. ${ }^{11}$ Due to a large $\Delta n$ value of $1.4 \times 10^{-2}$ (i.e., strong coupling) relatively short DBR regions $(70-100 \mu \mathrm{m})$ should be adequate. ${ }^{11}$ Furthermore, the DBR regions will be pumped over half their lengths, resulting in a relatively small penalty $(<10 \%)$ in overall efficiency.

Before presenting the results of the analysis involving DBR reflectors, we should define the important parameters. The gain threshold is defined as

$$
g_{\text {th }}=\alpha_{\text {rad }}+\alpha_{\text {edge }}-\kappa_{g} f_{\text {st }},
$$

where $\alpha_{\text {rad }}$ is the surface emission loss, $\alpha_{\text {edge }}$ is the edge emission loss, and $f_{\mathrm{st}}$ is the relative depth of the standing wave pattern. ${ }^{2}$ The external differential quantum efficiency is defined as

$$
\eta_{D}=\frac{\alpha_{\mathrm{rad}}}{g+\alpha_{i}},
$$

where $\alpha_{i}$ is the internal cavity loss, which is assumed to be 3 $\mathrm{cm}^{-1}$. Finally we define a parameter we call "aspect ratio" characterizing the longitudinal near-field profile of the surface emission. The aspect ratio is defined as the near-field Downloaded 29 Dec 2006 to 128.104.198.71. Redistribution subject

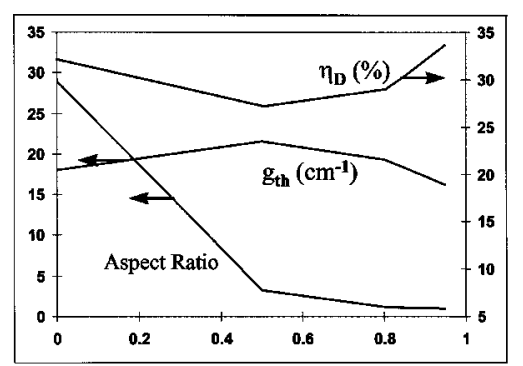

(a)
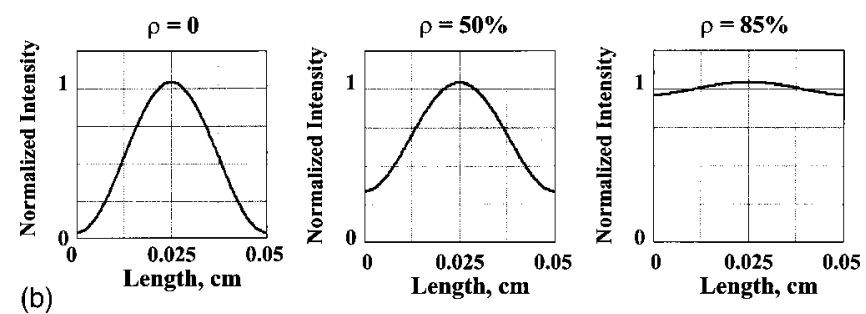

FIG. 3. For the structure shown in Fig. 2: (a) Gain threshold, $g_{\text {th }}$, external differential quantum efficiency, $\eta_{D}$, and aspect ratio of the symmetric mode as a function of DBR amplitude reflectivity, $\rho$; (b) near-field intensity profile of symmetric-mode surface emission for three values of $\rho: 0, \%, 50 \%$, and $85 \%$.

intensity at the center of the SE-CC-DFB divided by the near-field intensity at one end of the SE-CC-DFB (at the second-order/first-order grating interface). Thus, the aspect ratio quantifies the degree of nonuniformity of the near-field intensity profile - a large aspect ratio corresponds to a highly nonuniform near field while unity aspect ratio corresponds to a perfectly flat near-field intensity profile.

Figure 3(a) is a graphical representation of the results of the calculation of the aforementioned parameters for the structure of Fig. 2, and for a surface-emitting grating length of $500 \mu \mathrm{m}$. The graph shows, for the symmetric mode, how $g_{\text {th }}, \eta_{D}$, and the aspect ratio vary as the DBR amplitude reflectivity $\rho$ is varied from $0 \%$ to $95 \%$. Most notable is how quickly the aspect ratio approaches the ideal value of 1 . It is also notable that $g_{\text {th }}$ and $\eta_{D}$ vary relatively little with increasing $\rho$. Figure 3(b) shows the near-field intensity profiles for the cases when $\rho=0 \%, \rho=50 \%$, and $\rho=85 \%$. The reason why $g_{\text {th }}$ and $\eta_{D}$ vary so little with $\rho$ can be seen from Fig. 3 (b) and from Eqs. (1) and (2). The surface loss $\left(\alpha_{\text {rad }}=6.5\right.$ $\left.\mathrm{cm}^{-1}\right)$ and the absorption loss $\left(-\kappa_{g} f_{\mathrm{st}}=8.7 \mathrm{~cm}^{-1}\right)$ in the second-order DFB region are independent of $\rho$. Only the edge loss varies with $\rho$. In the first case, when $\rho=0$, there is almost no field at the ends of the SE-CC-DFB, and so the edge loss is small $\left(\alpha_{\text {edge }}=2.8 \mathrm{~cm}^{-1}\right)$. In the case where $\rho$ $=50 \%$, the field at the edges and therefore the edge loss is larger $\left(\alpha_{\text {edge }}=6.3 \mathrm{~cm}^{-1}\right)$. This causes only a relatively small increase in $g_{\text {th }}$ and decrease in $\eta_{D}$, since $\alpha_{\text {edge }}$ is relatively small compared with $\alpha_{\text {rad }}-\kappa_{g} f_{\text {st }}$. Finally, when $\rho=85 \%$, the field at the edges is large, but since the amplitude reflectivity is high, most of the field is reflected back into the structure and therefore, again the edge losses are small $\left(\alpha_{\text {edge }}=3.1 \mathrm{~cm}^{-1}\right)$; and $g_{\text {th }}$ and $\eta_{D}$ are back to what they were for $\rho=0$. The results show that one can drastically reduce the aspect ratio without paying a penalty in gain threshold or slope efficiency. That is, high near-field uniformity and high $\eta_{D}$ are simultaneously achievable. However, it should be noted that phase mismatches at the second-order/ to AIP license or copyright, see http://apl.aip.org/apl/copyright.jsp 


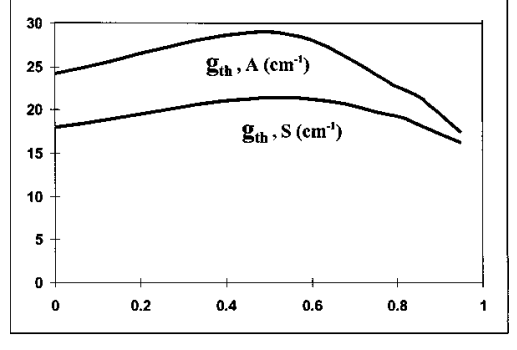

Amplitude Reflectivity, $\rho$

FIG. 4. Gain thresholds of the symmetric mode and the nearest antisymmetric mode as a function of the DBR amplitude reflectivity, $\rho$, for the structure shown in Fig. 2.

first-order grating interfaces can cause severe near-field distortions. We find that phase mismatches of up to $\pm \pi / 10$ are tolerable, in that the resulting near-field distortions are not enough to prevent symmetric-mode lasing to high drive levels. As mentioned above, e-beam grating fabrication has allowed successful operation of devices with second-order/ first-order grating interfaces. ${ }^{9,11}$ Should phase mismatches prove to be a problem the solution would be to use separate contact pads for the DBR reflectors so as to adjust the phase via carrier-induced changes in the dielectric constant.

Figure 4 shows how the gain thresholds of the symmetric mode, $g_{\text {th, }}$, and the nearest antisymmetric mode, $g_{\text {th }, \mathrm{A}}$, vary with $\rho$. The maximum modal discrimination occurs when $\rho=50 \%$. However the aspect ratio at that point is not adequate for high power single-mode operation. As $\rho$ approaches $100 \%$ the modal discrimination approaches zero and the aspect ratio approaches 1 . Therefore one must find a value of $\rho$ between $50 \%$ and $100 \%$ which provides suitable modal discrimination and low aspect ratio. For the device of Fig. 2, with a 500- $\mu \mathrm{m}$-long SE-CC-DFB section, we choose $\rho=85 \%$. This gives a modal discrimination value, $\Delta \alpha$, of $3.3 \mathrm{~cm}^{-1}$ and an aspect ratio of 1.1 ; while $g_{\mathrm{th}}=18 \mathrm{~cm}^{-1}$ and $\eta_{D}=30 \%$. The $\Delta \alpha$ value is considered adequate since the near-field profile for $\rho=85 \%$ [right-hand side of Fig. 3(b)] is virtually uniform and thus multimoding via longitudinal GSHB is highly unlikely. A simple single-spatial-mode structure in the lateral direction (e.g., ridge guide) together with the proposed structure should thus provide $\geqslant 100 \mathrm{~mW}$ cw surface-emitted, stable, single-mode power.

These results have important implications for the devel- opment of 2D high-power surface emitters. A ROW array is a structure for which all elements of a lateral array of antiguides equally couple with one another ${ }^{12}$ (i.e., parallel coupling) and thus the device has a uniform near-field intensity profile. ${ }^{12}$ The combination of the ROW array with the SECC-DFB with DBR reflectors will then result in a device with a uniform near field in both the lateral as well as the longitudinal directions; i.e., in two dimensions. The far field of such a device will be single lobed and normal to the surface. A device with a 20 -element ROW array $(\simeq 100 \mu \mathrm{m}$ aperture) and a 500- $\mu \mathrm{m}$-long SE-CC-DFB, being immune to GSHB and possessing strong (lateral) built-in index guiding, has the potential for providing greater than $1 \mathrm{~W} \mathrm{cw}$ stable, diffraction-limited power.

We have shown here that the addition of first-order DBR reflectors at the ends of a SE-CC-DFB laser drastically reduces the near-field nonuniformity, while the gain threshold and external differential quantum efficiency are relatively unaffected. Such devices should provide $>100 \mathrm{~mW}$ of stable power from (laterally) single-mode devices. Furthermore, by combining this structure with a ROW array one can create a two-dimensional surface emitter of uniform intensity profile, which has the potential of providing greater than $1 \mathrm{~W} \mathrm{cw}$ power in a stable, single-lobed beam pattern.

${ }^{1}$ K. David, J. Buus, and R. G. Baets, IEEE J. Quantum Electron. 28, 427 (1992)

${ }^{2}$ R. G. Baets, K. David, and G. Morthier, IEEE J. Quantum Electron. 29, 1792 (1993).

${ }^{3}$ J. Zoz, T. W. Johannes, A. Rast, B. Borchert, U. Barabas, and W. Harth, IEEE J. Quantum Electron. 31, 1432 (1996).

${ }^{4}$ T. Makino, H. Lu, and J. D. Evans, Appl. Phys. Lett. 68, 1892 (1996).

${ }^{5}$ A. Rast, T. W. Johannes, W. Harth, and G. Franz, IEE Proc.: Optoelectron. 142, 162 (1995).

${ }^{6}$ M. L. Osowski, R. Panepucci, I. Adesida, and J. J. Coleman, IEEE Photonics Technol. Lett. 9, 422 (1997).

${ }^{7}$ M. Kasraian and D. Botez, Appl. Phys. Lett. 69, 2795 (1996).

${ }^{8}$ M. Kasraian, J. Lopez, and D. Botez, IEEE Photonics Technol. Lett. 10, 27 (1998).

${ }^{9}$ N. Eriksson, M. Hagberg, and A. Larsson, IEEE Photonics Technol. Lett. 7, 1394 (1995).

${ }^{10}$ R. J. Noll and S. H. Macomber, IEEE J. Quantum Electron. QE-26, 456 (1990).

${ }^{11}$ M. Hagberg, N. Eriksson, and A. Larsson, IEEE J. Quantum Electron. QE-32, 1596 (1996).

${ }^{12}$ D. Botez, Diode Laser Arrays, edited by D. Botez and D. R. Scrifres (Cambridge University Press, Cambridge, UK, 1994), pp. 1-72. 